\title{
GENITOPLASTIA FEMINIZANTE EN HIPERPLASIA SUPRARRENAL CONGÉNITA; ¿UNA O DOS ETAPAS QUIRÚRGICAS?
}

\author{
José Manuel Escala Aguirre', Yair Cadena, Pedro-José López, Lorena Angel, María G. Retamal, \\ Nelly Letelier y Ricardo Zubieta.
}

Departamento de Urología Pediátrica. Hospital Exequiel González Cortés. Santiago. Chile.

'Profesor Asociado de la Universidad de Chile. Santiago. Chile.

\begin{abstract}
Resumen.- OBJETIVO: El mejor momento para realizar una genitoplastia en una niña con Hiperplasia Suprarrenal Congénita (HSRC) es un tema que ha sido debatido ampliamente. El objetivo de este estudio es encontrar un criterio que pueda ayudar en esta decisión.

MÉTODO: Se revisaron los datos de todos los pacientes con diagnóstico de HSRC con déficit de 21 Hidroxilasa que se sometieron a genitoplastia en nuestra institución (Enero 1996-Diciembre 2006). Se analizaron datos demográficos, cirugía realizada y resultados.
\end{abstract}

\section{CORRESPONDENCIA}

José Manuel Escala

Yair Cadena González

Departamento de Urología Pediátrica

Hospital Exequiel González Cortés

Barros Luco 3344 San Miguel

Santiago. (Chile).

jmescala@clc.cl

yaircadena@yahoo.com

Trabajo recibido: 22 de abril 2009.
RESULTADOS: En el periodo de 10 años, 25 pacientes cumplieron los criterios de inclusión; 22 tenían datos completos. Todos los pacientes fueron clasificados basándose en los criterios de Prader; Prader 2 ( $n=3)$, Prader $3(n=13)$ y Prader $4(n=6)$. El promedio de edad al momento de la primera cirugía fue 13.5 meses /rango 2-89m). En los pacientes Prader 2, una clitoroplastia de reducción con una vaginoplastia "cut back" fue realizada sin complicaciones. Todos los pacientes del grupo Prader 3 se sometieron a una clitoroplastia de reducción. Una vaginoplastia fue hecha en 9/13; 5/9 en la misma sesión quirúrgica (4 estenóticas) y los otros 4 en un segundo tiempo, con buenos resultados; en las otras 4 niñas la vaginoplastia está aún pendiente. En el grupo Prader 4, una vaginoplastia "pull-through" fue realizada en 4/6 usando un abordaje sagital posterior; uno al momento de la clitoroplastia de reducción, con resultados estenóticos y necesidad de dilataciones, y los otros 3 en una segunda cirugía con buenos resultados. Las otras 2/6 niñas están esperando una vaginoplastia. $22 / 22$ tiene resultados aceptables luego de un periodo de seguimiento de 63 meses (rango 12-144m).

CONCLUSIONES: La Hiperplasia Suprarrenal Congénita (HSRC) muestra diferentes grados de virilización, por lo tanto distintos abordajes pueden ser utilizados. Para los casos menos severos (Prader <3) un "cut-back" puede ser la cirugía de elección para una vaginoplastia, mientras en los casos más complejos un colgajo con "pull-through" o un procedimiento sagital posterior puede ser útil. Basado en esta serie, nosotros recomendamos realizar una vaginoplastia en un segundo tiempo quirúrgico, evitando complicaciones y procedimientos adicionales como las dilataciones. 
Palabras clave: Hiperplasia Suprarrenal Congénita (HSRC). Pediátricos. Clitoroplastia. Vaginoplastia.

Summary.- OBJECTIVES: The best time to perform a genitoplasty in a Congenital Adrenal Hyperplasia (CAH) girl is an issue that has been discussed extensively. The purpose of this study is to find criteria that may help in the decision.

METHODS: Charts of all patients with diagnosis of $\mathrm{CAH}$ with 21 Hydroxylase deficit who underwent genitoplasty in our institution were reviewed (Jan 1996-Dec 2006). Demographic data, surgery performed and outcomes were analyzed.

RESULTS: In the 10 year-period, 25 patients fit the inclu sion criteria; 22 had complete data. All patients were classified based on Prader's criteria; Prader $2(n=3)$, Pra der $3(n=13)$ and Prader $4(n=6)$. Mean age at first surgery was 13.5 months (range 2-89m). In Prader 2 patients, a reduction clitoroplasty with a "cut back" vaginoplasty was performed with no complications. All patients in the Prader 3 group underwent a reduction clitoroplasty. A vaginoplasty was done in 9/13;5/9 at the same surgery session (4 stenotic) and the other 4 in a 2nd stage with good results; vaginoplasty is still pending for the other 4 girls. In the Prader 4 group, a vaginoplas ty pull-through was performed in 4/6 using the poste rior sagital approach; one at the reduction clitoroplasty stage which ended stenotic and need dilatations, and the other 3 in a 2nd surgery with a good outcome. The other 2/6 girls are awaiting a vaginoplasty. 22/22 had acceptable results after a mean follow-up of 63 months (range 12-744).

CONCLUSIONS: Congenital Adrenal Hyperplasia (CAH) shows di fferent approaches may be used for different degrees of virilization. For less severe cases (Prader $<3$ l) a cut-back may be the surgery of choice for vaginoplasty, while in the more complex cases a flap with pull-through or a posterior sagital procedure could be useful. Based on this series, we recommend performing vaginoplasty in a 2 nd stage surgery, avoi ding complications and further procedures such as dilatations.

Keywords: Congenital Adrenal Hyperplasia (CAH). Paediatrics. Clitoroplasty. Vaginoplasty.

\section{INTRODUCCIÓN}

La hiperplasia suprarrenal congénita es generada por una serie de desórdenes en una de las cinco enzimas necesarias para la síntesis de cortisol en la corteza adrenal (1).
El desorden más común es el déficit de 21 hidroxilasa; éste está presente sobre el $90 \%$ de los casos. La deficiencia genera virilización en diferentes grados y es la causa más frecuente de ambigüedad genital $(1,2)$. Los objetivos quirúrgicos incluyen tres pasos principales en pacientes con virilización debido a hiperplasia suprarrenal: Reconstrucción del clítoris, del labio mayor y vaginoplastia en búsqueda de resultados estéticos y funcionales adecuados. La función vesical debe ser también adecuadamente preservada, así como la capacidad de desarrollar una adecuada vida sexual y reproductiva cuando adulto.

Basado en estas premisas, varias técnicas quirúrgicas han sido desarrolladas, donde el conocimiento de la anatomía, grado de virilización, longitud del seno urogenital y uretra, así como otras anomalías asociadas son esenciales $(3,4)$.

La clitoroplastia es generalmente recomendada en pacientes bajo los seis meses de edad, pero el momento ideal para realizar la vaginoplastia sigue siendo un debate $(1,2,5)$. Cuando el seno urogenital (SUG) es muy corto, la clitoroplastia y vaginoplastia puede ser realizada al mismo tiempo (2). Sin embargo, cuando la vagina llega más arriba en el seno urogenital, la vaginoplastia se podría hacer más adelante, para así obtener mejores resultados finales.

El objetivo de este trabajo es describir nuestra experiencia en ambas reconstrucciones, temprana y tardía, del seno urogenital, y analizar los resultados buscando los puntos de vista que podrían contribuir a alcanzar una mejor decisión acerca de un adecuado tiempo quirúrgico en los diferentes casos.

\section{MATERIALES Y MÉTODOS}

Este estudio es una revisión de las historias clínicas de los pacientes con hiperplasia suprarrenal congénita virilizante debido a déficit de 21 -hidroxilasa, quienes fueron tratados en nuestra institución entre 1996 y 2006.

El grado de virilización fue registrado en cada caso de acuerdo a Prader y la longitud del seno urogenital fue medida por cistoscopia. Los pacientes fueron divididos en tres grupos, de acuerdo al grado de virilización y longitud del seno urogenital. La edad de los pacientes fue registrada y la técnica quirúrgica empleada en la vaginoplastia fue analizada. Los resultados a largo plazo, complicaciones y necesidad de procedimientos adicionales también fueron registrados. 
TABLA I. DISTRIBUCIÓN INICIAL DE PACIENTES.

\begin{tabular}{|c|c|c|c|}
\hline Grupo & \# Pacientes & Prader & Promedio longitud seno (cm) \\
\hline 1 & 3 & 2 & 0.8 \\
\hline 2 & 13 & 3 & 1.6 \\
\hline 3 & 6 & 4 & 3.5 \\
\hline
\end{tabular}

\section{RESULTADOS}

Durante el periodo de 10 años hubo 25 pacientes que cumplieron los criterios para ser incluidos en este estudio, y los datos completos estaban disponibles en 22 de ellos. Los pacientes fueron divididos en tres grupos, considerando la escala de virilización de Prader y la longitud del seno urogenital. El grupo 1 (3 pacientes) incluyó pacientes con Prader 2 y longitud promedio de SUG de $0.8 \mathrm{~cm}$ (rango 0.5 a 1 $\mathrm{cm}$ ). Grupo 2 (13 pacientes) con Prader 3 longitud

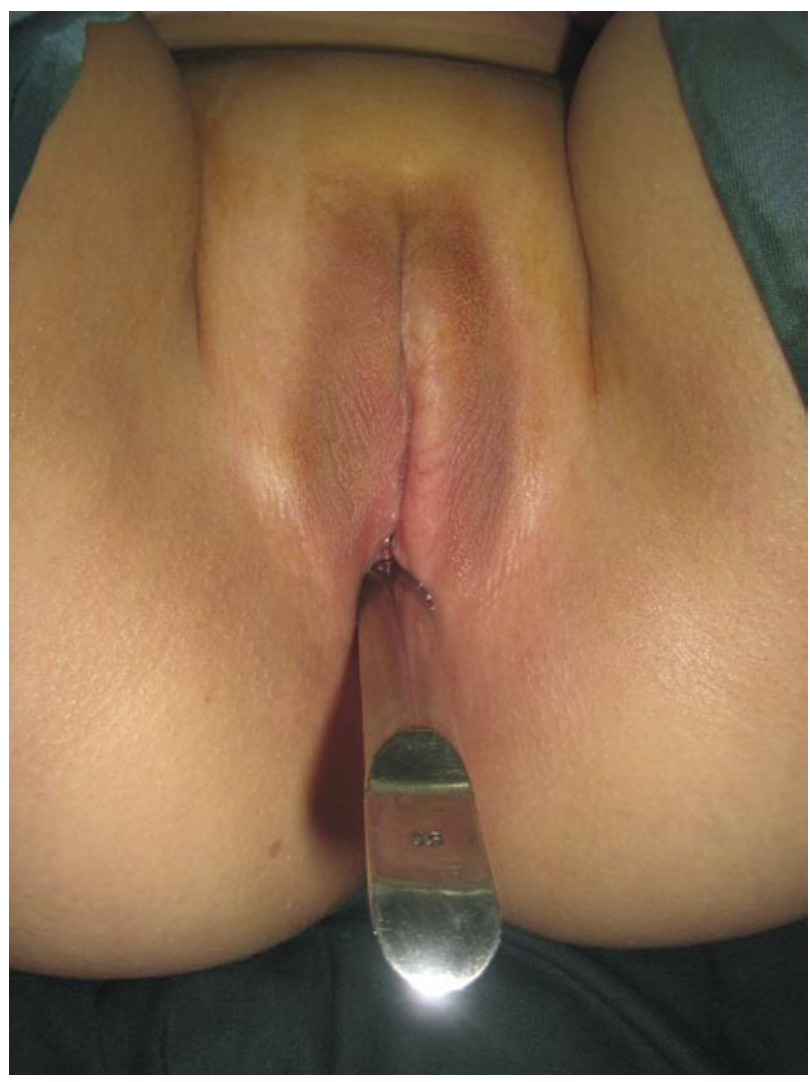

FIGURA 1. Aspecto estético a la edad de 12 años. Dilatación Hegar 14. promedio de SUG de $1.6 \mathrm{~cm}$, (rango $1.3 \mathrm{a} 2 \mathrm{~cm}$ ). Grupo 3 (6 pacientes) con Prader 4 y longitud promedio de $S U G$ de $3.5 \mathrm{~cm}$, (rango 3 a $4 \mathrm{~cm}$ ) (Tabla I).

Todos los pacientes tuvieron un chequeo endocrinológico complete, estudio urológico, ecografía renal y del tracto urinario, uretrocistografía y fueron examinados bajo anestesia.

La clitoroplastia fue hecha en un primer estadío quirúrgico en todos los pacientes, con resección de los cuerpos cavernosos y conservación del paquete vásculo-nervioso. El promedio de edad al momento del primer procedimiento fue 13.5 meses en los tres grupos (2- 89 meses) y no hubo diferencia significativa entre los grupos, respecto a los resultados y complicaciones.

En el primer grupo, la vaginoplastia fue realizada en el mismo tiempo quirúrgico que la clitoroplastia y en los tres casos se realizó utilizando la técnica "cut back". No hubo complicaciones en

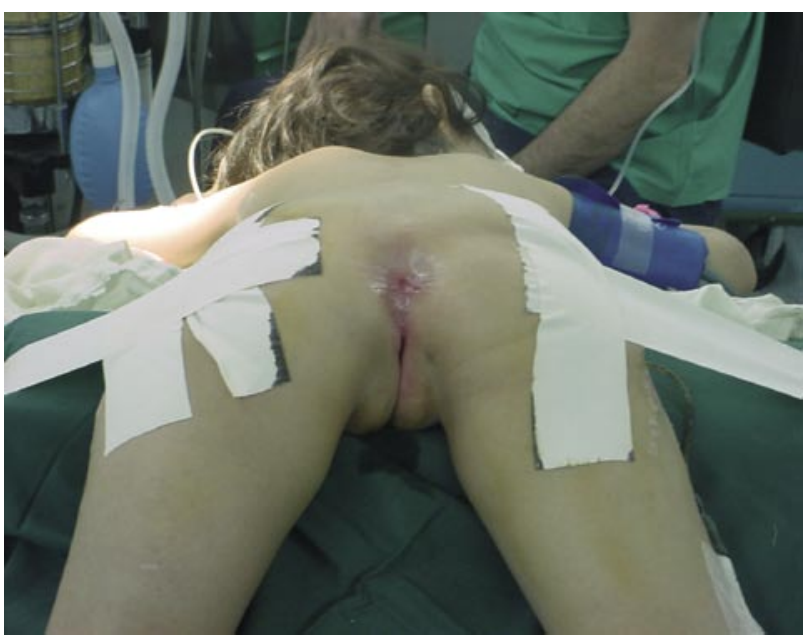

FIGURA 2. Posición del abordaje sagital posterior. 


\section{TABLA II. RESULTADOS DESPUÉS DE 63 MESES (PROMEDIOI DE SEGUIMIENTO.}

\begin{tabular}{|c|c|c|c|}
\hline Prader/promedio longitud seno & Promedio edad vaginoplastia & Pacientes & Buenos resultados \\
\hline $2 / 0.8 \mathrm{~cm}$ & 22 meses & 3 & $3(100 \%)$ \\
\hline \multirow{2}{*}{$3 / 1.6 \mathrm{~cm}$} & 9 meses & 5 & $1(20 \%)$ \\
\cline { 2 - 4 } & 108 meses & 4 & $4(100 \%)$ \\
\cline { 2 - 4 } & Pendiente & 1 & - \\
\hline \multirow{2}{*}{$4 / 3.5 \mathrm{~cm}$} & 26 meses & 3 & $3(100 \%)$ \\
\cline { 2 - 4 } & 156 meses & 2 & - \\
\cline { 2 - 4 } & Pendiente & & 0 \\
\hline
\end{tabular}

ninguno de los casos; todos los pacientes mostraron una apariencia estética aceptable, y no necesitaron procedimientos adicionales.

En el grupo 2, 5/13 vaginoplastia se realizaron en el mismo tiempo quirúrgico que la clitoroplastia. En estos casos, la vaginoplastia fue realizada usando un colgajo en $U$ invertido de Fortunoff y movilización parcial del seno. De estos 5 pacientes, 4 presentaron estenosis vaginal, que requirieron dilataciones, y en 3 de los 4 casos un nuevo procedimiento quirúrgico está en espera de ejecución, debido a que el programa de dilatación falló. Un resultado satisfactorio a largo plazo se logró en un sólo paciente, con un resultado estético aceptable y un adecuado calibre vaginal. En otros cuatro pacientes de este grupo, la vaginoplastia se pospuso para un segundo tiempo quirúrgico a una edad promedio de 108 meses (rango 84-1 14 meses). La misma técnica de colgajo en $U$ invertido y movilización parcial del seno fue empelada. Un adecuado resultado estético y funcional se logró en los cuatro casos (Figura 1). La vaginoplastia está pendiente en 4/13 pacientes en este grupo.

En el grupo 3, y en uno de seis pacientes, la vaginoplastia fue realizada al mismo tiempo quirúrgico que la clitoroplastia, usando un desplazamiento en bloque por via sagital psoterior $(2,3,4)$. Aunque este paciente mostró una adecuada recuperación, fue necesario realizar dilataciones vaginales. En otros 3 pacientes de este grupo la vaginoplastia fue realizada en un segundo tiempo quirúrgico, con el mismo abordaje, dando resultados satisfactorios en lo que respecta a la apariencia, continencia y calibre vaginal. La vaginoplastia está aún pendiente en 2 pacientes de este grupo (Tabla II).

Ninguno de estos pacientes desarrolló incontinencia urinaria y todos tienen una buena apariencia estética. Al momento, 6 pacientes están esperando una vaginoplastia en un segundo tiempo quirúrgico.

\section{DISCUSIÓN}

La hiperplasia suprarrenal congénita puede causar virilización en diferentes grados, por lo tanto diferentes enfoques deben ser empleados para resolver quirúrgicamente estas alteraciones, dependiendo de su severidad (1).

Aunque han sido descritas muchas técnicas quirúrgicas para abordar el seno urogenital, con resultados variados, el mejor momento para realizar la reconstrucción vaginal continúa siendo un debate.

En la reconstrucción genital, la clitoroplastia ha sido clásicamente realizada a una edad muy temprana, para así obtener un resultado estético adecuado. Por otro lado, se argumenta que la reconstrucción sería mucho más fácil a esa edad, debido a la mayor movilidad de los tejidos, permitiendo una mejor disección (2).

Otro punto a favor de la reconstrucción temprana es aliviar la ansiedad de los padres y ayudar en la construcción de un rol de mujer para la pa- 
ciente desde el nacimiento (6). Sin embargo, algunos estudios informan que habría pacientes con disconformidad de su genero asignado, por lo que algunos autores sugieren que estos procedimientos deberían ser retrasados hasta que el paciente tenga una identidad sexual definida $(7,8)$.

En nuestra experiencia, preferimos que la clitoroplastia se realice en forma temprana, para así proporcionar una identidad propia desde una edad temprana, primero en los padres y más tarde en el paciente. Además, la reconstrucción del clítoris es técnicamente más sencilla a esta edad $(2,6,7,8)$.

En cuanto a la vaginoplastia, nuestra experiencia muestra como, con diferentes grados de virilización y longitud del seno urogenital, hemos llegado a diferentes resultados (Tabla II). Así, para pacientes con SUG corto $(<1 \mathrm{~cm})$ la clitoroplastia y vaginoplastia fue realizada en una sola etapa quirúrgica, realizando un corte posterior, tipo "cut-back" y obteniendo muy buenos resultados finales en esta serie.

En pacientes con un mayor grado de virilización (Prader 3, o longitud del seno $>1.3$ a $2 \mathrm{~cm}$ ), los abordajes han incluido realizar una movilización parcial del seno asociado a un colgajo de Fortunoff $(9,10)$. En estos casos el seguimiento ha mostrado que hay una mejor evolución en pacientes en quienes el procedimiento es realizado en un segundo tiempo quirúrgico, más que a edades más tempranas.

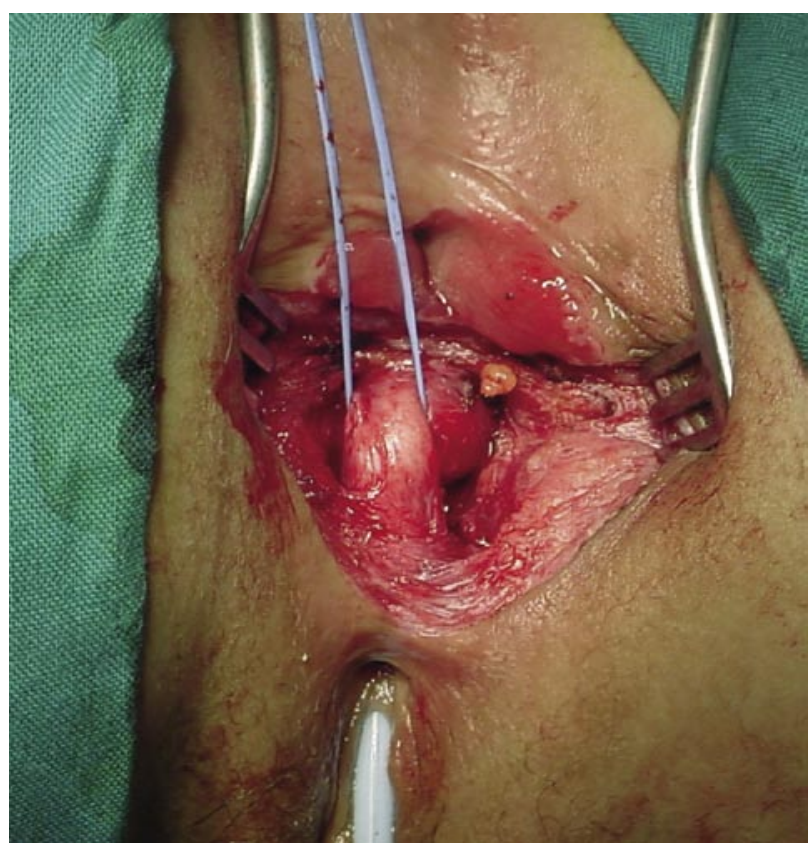

FIGURA 3. Disección vaginal, abordaje sagital posterior.
Estos resultados podrían ser explicados por el incremento hormonal en estos pacientes a esa edad, lo que aumentaría la maleabilidad de los tejidos y su vascularización (2). Por otro lado, la menstruación podría ayudar a mantener la permeabilidad del canal, por lo tanto, sumado a los cambios hormonales, esto podría dar un mejor resultado final al procedimiento (2). El hecho que pacientes mayores pueden colaborar con el manejo post operatorio, realizando dilataciones si fuera necesario o usando tampones vaginales, también podría influir en los resultados.

Sin embargo, otros estudios muestran que resultados adecuados pueden ser obtenidos en edades más tempranas $(2,11)$. Gosalbez et al (3) realizó una movilización de el SUG en estos casos, sustituyendo el uso de colgajo de Fortunoff, por un colgajo obtenido desde el SUG mismo.

En este estudio, a la edad promedio de 3.8 años y mostrando buenos resultados al momento de la evaluación, la mayor importancia se da a la longitud uretral para el procedimiento. Como nuestro estudio es retrospectivo, desafortunadamente este preciso dato no fue registrado en todos los casos.

En SUG de mayor longitud $(>3 \mathrm{~cm})$ y mayor grado de virilización (Prader 4), nosotros realizamos un abordaje sagital posterior descrito por Peña

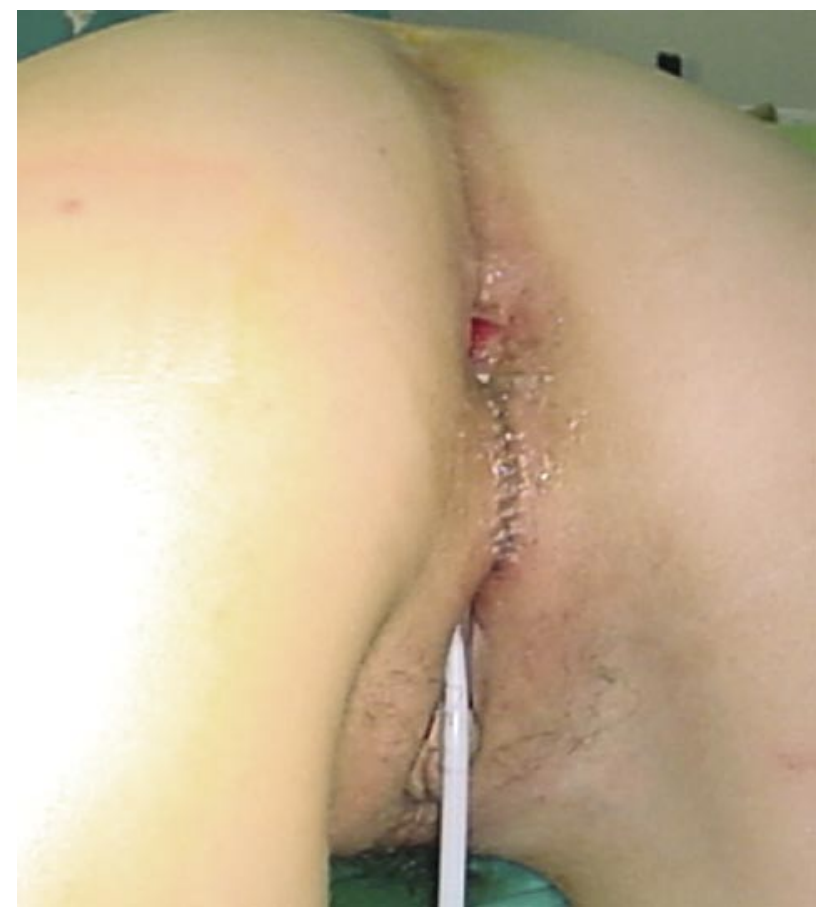

FIGURA 4. Resultado final del abordaje sagital posterior. 
$(12,13)$. Aunque este abordaje fue descrito primero para anomalías de tipo cloacal, con movilización total $(12,13)$, desde entonces uno de los autores principales (R.Z) ha promovido este abordaje para otras anomalías y problemas urológicos; los resultados en SUG en estas series son comparables a los de la literatura $(2,3,4,10,11)$. Este abordaje permite una gran exposición del campo operatorio y facilita una adecuada movilización. Un paciente de este grupo, en quien la vaginoplastia fue realizada al mismo tiempo que la clitoroplastia, mostró un buen aspecto, aunque fue necesario realizar dilataciones vaginales debido a que era demasiado estrecho.

La principal crítica que se hace a esta técnica es que podría provocar incontinencia urinaria $(2$, 12). Esto no ha ocurrido hasta la fecha en nuestras series, además, se ha visto que en el tiempo y con entrenamiento vesical el riesgo de incontinencia disminuye (14). Además la preservación de el ligamento puborectal, tanto en movilización total y parcial, da mejores resultados en incontinencia (10).

Así, los resultados a largo plazo muestran que el principal problema de los pacientes sometidos a vaginoplastia temprana es el estrechamiento de la vagina (5/16 en nuestras series), esto interfiere con la actividad sexual de la paciente, por lo tanto ha sido recomendado realizarlo en una segunda fase, en el inicio de la pubertad, para prevenir estenosis vaginal y si las dilataciones fuesen necesarias, poder realizarlas adecuadamente.

Para este mismo tipo de pacientes, es también posible realizar una movilización completa del SUG, que puede ser realizada por un abordaje perineal aunque a edades más avanzadas que la clitoroplastia, existen datos publicados con movilizaciones a la edad promedio de 4 años y con buenos resultados finales (4); esto también ha sido realizado en pacientes de menos de 3 años y 5,9 años $(16,17)$.

Por otro lado, y siendo conciente que el resultado estético es difícil de evaluar, los resultados en nuestros pacientes son aceptados por parte de las propias niñas, sus padres y el equipo quirúrgico.

A pesar de todos los procedimientos realizados y el progreso en el manejo de la patología, donde se busca un buen resultado estético y funcional, algunos estudios a largo plazo $(5,7,15)$ muestran que en pacientes sometidos a genitoplastia feminizante debido a hiperplasia suprarrenal congénita, los resultados son desalentadores, ya que los pacientes muestran disfunción sexual, disminución de la sensibilidad y resultados estéticos desfavorables, por lo tanto hay tendencias que prefieren posponer todos los procedimientos para una etapa posterior (5).

En nuestra práctica, preferimos realizar la clitoroplastia a una edad temprana, si es posible antes de los 6 meses, y la vaginoplastia en forma posterior, cuando las condiciones sean más adecuadas para ello.

\section{CONCLUSIONES}

El mejor momento para la vaginoplastia en hiperplasia suprarrenal congénita continúa siendo un debate. Basado en los resultados de esta serie, preferimos realizar la clitoroplastia a una edad temprana, junto con la vaginoplastia en los casos menos severos, como Prader 2 o SUG menor de $1 \mathrm{~cm}$.

Para los pacientes con mayor grado de virilización y/o longitud SUG $>3 \mathrm{~cm}$, preferimos realizar la vaginoplastia por un abordaje sagital posterior en una segunda etapa, para obtener mejores resultados estéticos y funcionales y prevenir realizar procedimientos adicionales.

\section{BIBLIOGRAFÍA y LECTURAS RECOMENDADAS (*lectura de interés $y^{* *}$ lectura fundamental)}

*1. Phyllis W, Speiser MD, and Perrin C. White. Congenital Adrenal Hyperplasia. N Engl J Med, 2003; 349:776-88.

2. Braga L, Lorenzo A J, Tatsuo E S, Silva I N and Pippi Salle J L. Prospective Evaluation of Feminizing Genitoplasty Using Partial Urogenital Sinus Mobilization for Congenital Adrenal Hyperplasia. J Urol, 2006; 176: 2199-2204.

**3. Gosalbez R, Castellan M, Ibrahim E, Disandro $\mathrm{M}$ and Labbie A. New concepts in feminizing genitoplasty-is the Fortunoff flap obsolete? J. Urol, 2005; 174: 2350-2353.

4. Ludwikowski B, OeschHayward I and González R. Total urogenital sinus mobilization: expanded applications. BJU Int,1999; 83: 820-822

5. Naomi S. Crouch and Sarah M. Creighton. Long-term functional outcomes of female genital reconstruction in childhood. BJU Int, 2007; 100(2):403-7.

6. Clayton P E, Miller W L, Oberfield S E, Ritzen E M, Sippell W G and Speiser P W. Consensus statement on 21-hydroxylase deficiency from the Lawson Wilkins Pediatric Endocrine Society and the European Society for Pediatric Endocrinology. J Clin Endocrinol Metab, 2002; 87: 4048. 
**7. Creighton S M. Long-term outcome of feminization surgery: the London experience. BJU Int, 2004; 93: 44.

8. Minto C L, Liao L M, Woodhouse C R, Ransley P G and Creighton S M. The effect of clitoral surgery on sexual outcome in individuals who have intersex conditions with ambiguous genitalia: a cross-sectional study. Lancet, 2003; 361: 1252.

9. Fortunoff S, Lattimer J K and Edson M. Vaginoplasty technique for female pseudohermaphrodites. Surg Gynecol Obstet, 1974; 118: 545

10. Rink R C, Metcalfe P D, Kaefer M A, Casale A J, Meldrum K K, Cain M P. Partial urogenital mobilization: A limited proximal dissection. Journal of Pediatric Urology,2006; 2: 351-356

*11. Rink R C, Metcalfe P D, Cain M P, Meldrum K K, Kaefer M A and Casale A J. Use of the Mobilized Sinus With Total Urogenital Mobilization. J. Urol, 2006; 176: 2205-2211.

**12. Pena A. Total urogenital mobilization - an easier way to repair cloacas. J Pediatr Surg, 1997; 32: 263.
**13. Pena A, Levitt MA, Hong A, Midulla P. Surgical management of cloacal malformations: a review of 339 patients. J Pediatr Surg, 2004; 39:470.

14. Kryger J V and González R. Urinary continence is well preserved after total urogenital mobilization. J Urol, 2004; 172, 2384-2386.

15. Krege $S$, Walz K H, Hauffa B P, Koèrner I and Ruèbben $\mathrm{H}$. Long-term follow-up of female patients with congenital adrenal hyperplasia from 21-hydroxylase deficiency, with special emphasis on the results of vaginoplasty. BJU International, 2000; 86: 253-259

16. Alaa F. Hamza, Hesham A. Soliman, Sameh A. Abdel Hay, Ashraf A. Kabesh, and Mossad M. Elbehery. Total Urogenital Sinus Mobilization in the Repair of Cloacal Anomalies and Congenital Adrenal Hyperplasia. J Pediatr Surg, 2001; 36:1656-1658.

*17. Roman Jenak, Barbara Ludwikowski and Ricardo Gonzalez. Total urogenital sinus mobilization: a modified perineal approach for feminizing genitoplasty and urogenital sinus repair. J Urol Vol, 2001; 165: 2347-2349. 